\title{
Development of Memory and the Hippocampus: Comparison of Food-Storing and Nonstoring Birds on a One-Trial Associative Memory Task
}

\author{
N. S. Clayton \\ Department of Zoology, Oxford University, Oxford OX1 3PS, United Kingdom
}

Food-storing birds, for example, marsh tits, Parus palustris, use memory to retrieve stored food and have a larger hippocampus relative to the rest of the telencephalon than do species that store little or no food such as the blue tit, $P$. caeruleus. The difference between food storers and nonstorers in relative hippocampal volume occurs after the young birds have fledged from the nest and is dependent upon some aspect of memory for retrieving caches of stored food. To test whether or not species differences in memory and volumetric changes in the hippocampus could be triggered by experience of memory tasks other than retrieval of stored food, groups of hand-raised marsh tits and blue tits were tested between days 35 and 192 posthatch on a one-trial associative memory task in which they were rewarded in phase II for returning to the feeder where they had eaten part of a peanut 20 min earlier. No species differences were found when the peanut was visible in phase $I$, but when the peanut was hidden in phase I, marsh tits performed better than blue tits, irrespective of whether or not they had had previous experience of storing and retrieving food. In dissociation trials (transformed array of feeders), marsh tits with food-storing experience responded preferentially to spatial cues, whereas blue tits responded equally to both spatial position and object-specific cues. These species differences are also found in wild-caught adults. However, marsh tits without food-storing experience responded equally to both spatial position and objectspecific cues, which suggests that experience of storing and/or retrieving caches is required in order for marsh tits to develop the spatial preference seen in adult food storers. Both marsh tits with experience of the one-trial associative memory task and those that had also had foodstoring experience had larger relative hippocampal volumes than did controls, independent of age. Of the marsh tits trained on the one-trial associative memory task,

\footnotetext{
Received June 27, 1994; revised Sept. 14, 1994; accepted Oct. 18, 1994.

This research was supported by a grant from the Agriculture and Food Research Council and a grant from the Science and Engineering Research Council. Hans-Joachim Bischof, Rob Hampton, John Krebs, and Sara Shettleworth critically commented on the manuscript. I also thank Jeremy Blakey, Jenny Corrigan, Matthew Evans, Andy Gosler, Rob Hampton, Angus Jackson, Annd Jolliffe, Gudrun Kabicher, Ryan Mellor, Tim Jones, Nick Lewis, John Muddeman, Alun ap Rhisiart, Sanjay Patel, Chris Perrins, Yu Tzu Tai, Paul Strecker Andrea Szekely, Ben Underwood, and Dave Wilson for technical assistance and Jonathan Newman for statistical advice.

Correspondence should be addressed to N. S. Clayton, Department of Zoology, South Parks Road, Oxford University, Oxford OX1 3PS, United Kingdom.

Copyright $(\mathcal{C} 1995$ Society for Neuroscience $\quad 0270-6474 / 95 / 152796-12 \$ 05.00 / 0$
}

there was no difference between those that had had foodstoring experience and those that had not. However, in blue tits, there was no effect of experience on relative hippocampal volume. No volumetric differences were observed in ectostriatum, which served as a control brain region. The results suggest that some aspect of memory for retrieving food (whether or not stored by the bird) directly influences growth of the hippocampal region in marsh tits, the food-storing species, but not in blue tits, the nonstoring species.

[Key words: avian, hippocampus, memory, food-storing birds]

One of the central questions in neuroscience concerns the relationship between memory and the hippocampus. Although most of the previous studies have been carried out on mammals,

Food-storing animals have an accurate, long-lasting memory on which they rely to retrieve their caches of stored food (review in Vander Wall, 1990). They also have a larger hippocampus (dorsomedial cortex) relative to the rest of the telencephalon than do nonstorers (review in Sherry et al., 1992). Lesions to this region suggest that the avian hippocampus plays a role in spatial memory, including the memory for cache sites in foodstoring birds (e.g., Krushinskya, 1966; Sherry and Vaccarino, 1987). These findings have led to the suggestion that food-storers have a specialized memory ability and associated neural substrates in the hippocampus (reviewed by Krebs, 1990; Shettleworth, 1990).

In order to test whether or not food-storing species have a memory ability that differs in quality or quantity from that of species which store little if any food, their performance should be compared in a task that does not involve storing food, but that reveals the essential features of memory used by food-storing species to retrieve their hoards. One such task is the "onetrial associative memory" test (Brodbeck et al., 1992; Clayton and Krebs, 1992) and the related procedure of "window-shopping" (Krebs et al., 1990; Shettleworth et al., 1990). In phase 1 , the bird searches for and eats part of a peanut located in one of a number of potentially trial-unique sites. In phase 2 the bird's memory performance is tested by allowing it to search for the remainder of the peanut. Accuracy of memory for the location of the partially eaten hidden food is measured by the number of errors (visits to other sites) before finding the peanut: fewer errors implies more accurate performance. The task resembles retrieval of stored food in that it the bird has to return to the rewarded site after a single previous experience of the peanut's location. It differs from food storing in that the bird does not 
place the peanut in the site and, in phase 2 , the bird has to remember a site where it obtained a reward previously rather than a site which contains a reward.

Using this task, the results of a number of studies have suggested that several food-storing species in both the Paridae and Corvidae differ from closely related species that store little or no food in some aspect of spatial memory. For example, Brodbeck (1994) and Clayton and Krebs (1994a) showed that the food-storing species preferentially respond to spatial cues when position and object cues are dissociated by transforming the array, while the nonstoring species respond equally to both kinds of cue. The results of a second experiment by Clayton and Krebs (1994b) suggested that food-storing species differ from nonstoring species in the way they discriminate between remembered events. When the peanut is hidden in phase I so that the bird has to search for the reward in both phases of a trial (hidden version), the food-storing species preferentially returned to the rewarded site, whereas the nonstoring species preferentially returned to sites that they had visited in phase $I$, irrespective of whether or not they contained a reward. However, when the reward was visible in phase 1 but hidden in phase II (visible version) and therefore the only class of visited sites is rewarded ones, no species differences in performance were found. These results can also be encompassed within the framework of spatial memory differences by interpreting them as showing that foodstoring species encode spatial information more accurately or in more detail than do species that store little or no food and hence are able to return to the rewarded site, as opposed to the more general class of visited sites.

It has been suggested that the differences between food storers and nonstorers in performance on the above tasks may be a reflection of differences in some aspect of spatial memory and that this may be associated with differences in the two species' relative hippocampal volumes (Clayton and Krebs, 1994b). A number of experiments have shown that the avian hippocampus, like the rat and primate hippocampus (O' Keefe and Nadel, 1978; Squire, 1993), plays a role in processing and/or storing spatial memory. For example, hippocampal damage has been shown to impair the ability of homing pigeons to navigate home from novel release sites within the familiar area around the loft (Bingman, 1990), retrieval of stored food (Sherry and Vaccarino, 1989), spatial discrimination learning (Sherry and Vaccarino, 1989), and spatial delayed nonmatching to sample (Good and Macphail, 1993). In contrast, at least some nonspatial tasks, such as color discrimination learning (Sherry and Vaccarino, 1989; Rice, 1992), delayed matching to sample with repeated stimuli and delayed nonmatching to sample with trial unique stimuli (Good and Macphail, 1993) are unimpaired by hippocampal damage. Thus, the relatively larger hippocampal volume found in food-storing species is at least consistent with the observed differences in spatial memory.

The difference between food-storing and nonstoring species in hippocampal volume arises at a relatively late stage in development, after the young birds have fledged from the nest (Healy and Krebs, 1993; Healy et al., 1994) and an obvious question is whether or not the species differences in performance on these memory tasks coincide with the increase in the food storer's relative hippocampal volume. Recent work on the dual ontogeny of the hippocampus and food-storing memory in marsh tits, Parus palustris, suggests that the relative increase in hippocampal volume is dependent upon some aspect of the experience of storing and retrieving food caches and that changes in both the behavior and brain can occur rapidly over a period of just $24 \mathrm{~d}$ (Clayton, 1992, 1994; Clayton and Krebs, 1994c).

Since lesions to the hippocampus disrupt memory-based retrieval but have no effect on food-storing behavior (Sherry and Vaccarino, 1989), the implication from these results is that the volumetric changes in the hippocampus are dependent on memory for cache sites rather than on the ability to store food per se. This raises the question of whether or not the volumetric changes in the hippocampus in marsh tits can be triggered by experience of memory tasks other than retrieval of stored food, such as training on the one-trial associative memory task and if so, whether or not experience-dependent changes could be triggered in the hippocampus of a species that stores little or no food such as the blue tit.

The aim of this study is to compare the ontogeny of memory and the hippocampus in food-storing marsh tits and nonstoring blue tits. The first objective is to test whether the observed differences between adult food-storing and nonstoring species in performance on the one-trial associative memory task are apparent in the juveniles. In order to do this, juvenile marsh tits and blue tits are trained on either the hidden $\mathrm{MH}$ and $\mathrm{BH}$, respectively) or the visible version (MV and $\mathrm{BV}$, respectively) of the test and subsequently given a series of dissociation trials. If the observed behavioral differences between adult food-storing and nonstoring species (Clayton and Krebs, 1994a,b) are apparent in juveniles then there should be no species differences in performance on the visible version but marsh tits should outperform blue tits on the hidden version; and in dissociation trials, marsh tits should prefer to return to the feeder in the correct spatial location whereas blue tits should prefer the correct spatial location on about $50 \%$ of trials and the correct feeder on about $50 \%$ of trials.

Birds are tested from the time at which they become nutritionally independent (day 35 posthatch) in order to examine whether or not performance on the one-trial associative memory task shows the same gradual increase as memory for retrieving caches of previously stored food. To determine the relative roles of age and food-storing experience the behavioral performance of the two marsh tit groups $\mathrm{MH}$ and MV were compared with two additional groups of marsh tits, MSH and MSV, which were given the opportunity to store and retrieve food for $60 \mathrm{~d}$ prior to the tests. If experience of food-storing memory is crucial then performance of birds in groups MSH and MSV should differ from those in groups $\mathrm{MH}$ and $\mathrm{MV}$ whereas if age is the key factor then there should be no difference between groups MSH, MSV, MH, and MV when tested at the same age.

The second objective is to test whether or not the volumetric changes in relative hippocampal size can be triggered by training birds on the one-trial associative memory task rather than by experience of storing and retrieving caches of food. Comparison of the relative hippocampal volumes between the groups of marsh tits tests whether or not the volumetric changes in relative hippocampal size can be triggered by training marsh tits on the one-trial associative memory task rather than by experience of food-storing and retrieval.

Two predictions can be made which follow from the suggestion that food-storing and nonstoring species may differ in some aspect of spatial memory which may be associated with differences in the two species' relative hippocampal volumes (Clayton and Krebs, 1994b). First, it is predicted that birds with larger relative hippocampal volumes should prefer to return to the correct spatial location in dissociation trials. Second, the relative 
Table 1. Stage of experiment (age, days posthatch)

\begin{tabular}{llll} 
Group & I (35-95) & II (152-192) & No. brains \\
\hline MH $(N=6)$ & Hidden task & Hidden task & 4 \\
MV $(N=6)$ & Visible task & Visible task & 4 \\
$\quad(1$ died during stage I) & Hidden task & Hidden task & 4 \\
BH $(N=6)$ & Visible task & Visible task & 4 \\
BV $(N=6)$ & Food-storing & Hidden task & 4 \\
$\quad(1$ died during stage I $)$ & Food-storing & Hidden task & 4 \\
MSH $(N-6)$ & Control & Control & 4 \\
$(1$ died during stage II $)$ & Control & Control & 4 \\
MSV $(N=6)$ & $(2$ died during stage II $)$ & & 4 \\
MC $(N=6)$ & BC $(N=6)$ & &
\end{tabular}

hippocampal volumes of birds trained on the hidden version should be larger than those trained on the visible version.

In order to test whether or not the changes in hippocampal volume can also be detected in a species that does not store food, blue tits that had experience on the one-trial associative memory task are also compared with age controls that lacked this experience. This is particularly important in determining whether or not there is something unique about the hippocampus of a food-storing species and therefore whether or not foodstoring species have an anatomical specialization of the brain which is associated with their memory for cache sites.

\section{Materials and Methods}

Birds, groups, and housing conditions. The subjects were 38 handraised, postfledging juvenile marsh tits, 21 hand-raised, postfledging juvenile bluc tits, and 4 wild-caught adult ( $>1$ year posthatch) blue tits which were taken from Wytham woods under NCC license. The juveniles were taken at day 12 posthatch as half broods from eight marsh tit and four blue tit clutches and hand-raised until day 35 (nutritional independence).

The birds were housed individually indoors (10:14 L:D cycle) in wire cages measuring $0.44 \mathrm{~m}$ wide $\times 0.77 \mathrm{~m}$ long $\times 0.44 \mathrm{~m}$ high and divided into groups. The birds from each group were matched for weight and clutch and were exposed to different kinds of behavioral experience as summarized in Table 1 . There were eight groups, each consisting of six individuals. The six experienced groups received daily trials on the onetrial associative memory task in which the birds obtained a reward in phase II by returning to the feeder where they had eaten part of the reward $20 \mathrm{~min}$ earlier in phase 1 . In the visible version, peanut was visible in phase 1 whereas in the hidden version peanut was hidden in phase 1; in both versions peanut was hidden in phase 2 to test for memory in relocating the partially eaten food (Clayton and Krebs, 1994b). The two control group also received daily trials which were identical to those of the experienced groups except that none of the feeders contained food and there was a bowl containing powdered peanut on the floor which the birds could eat. Thus, the birds flew and perched on the feeders but did not search in them because none of the feeders were baited. The duration of the two phases for the control groups were matched with those for the experienced groups.

Experimental environment. Birds were tested individually in a room, $3.5 \times 2.0 \times 2.8 \mathrm{~m}$ high, and observed through a one-way Plexiglas window. The room contained seven "peanut feeders" (Krebs et al., 1990) which were hung from plastic mesh screens measuring $1 \times 2 \mathrm{~m}$ attached to two adjacent walls of the room. Lach peanut feeder consisted of a wooden block measuring $9.0 \times 4.0 \times 15.0 \mathrm{~cm}$ high with a sloping top. In the center of the face of each wooden block there was a hole $0.5 \mathrm{~cm}$ diameter $\times 0.5 \mathrm{~cm}$ deep which could contain a piece of powdered peanut. This hole could be covered by a knot at the end of a short piece of string attached to the block to prevent the birds from seeing the food without first removing the knot (Clayton, 1992a; Hampton and Sherry, 1994); $4 \mathrm{~cm}$ below the hole there was a perch, $5.5 \mathrm{~cm}$ $\times 0.9 \mathrm{~cm}$ diameter, on which the bird could perch and examine the contents of the hole. The birds readily learned to pull the knot of string away from the hole to inspect the contents on trials when the food was hidden. Each feeder was made visually unique on every trial by addition or removal of colored adhesive paper shapes drawn from a very large pool. Although individual shapes were used on more than one occasion, the combinations were unique. For each trial, the location of the feeders was chosen at random from 400 possible coordinates on the two mesh walls (the array always included both walls) so that both spatial and object-specific site cues were trial-unique (Brodbeck et al., 1992; Brodbeck, 1994; Clayton and Krebs, 1994a). The floor was swept between each phase and trial to ensure there were no extraneous pieces of food in the test room.

Training. To ensure that all birds were familiar with the environment prior to the experiment all birds were given exposure to peanut feeders and the testing room for the period between fledging and independence (days 21-34). During the initial familiarization stage (days 21-27), birds were let into the room for $20 \mathrm{~min}$ daily. There was one feeder which hung on one of the walls. The birds also were given a feeder in their home cage and this was changed daily. In the control groups the feeders were not baited whereas in all the experienced groups the feeders contained powdered peanut which was either visible (MV, BV, and MSV) or hidden by the string knot (MH, BH, and MSH). During the second stage of training (days 28-34), seven feeders were placed in the room. For control birds the feeders were never baited but a bowl of powdered peanut was placed on the floor so that the birds could eat. For experienced birds, all seven feeders contained powdered peanut. Once the birds had learned to visit sites containing food, only one site was rewarded on each trial. Trial-unique sites were introduced at this stage for both experienced and control groups.

Undissociated trials. Each trial consisted of two phases, separated by a retention interval of $20 \mathrm{~min}$. In phase I, the bird searched for powdered peanut in one of seven sites and was allowed to eat for $10 \mathrm{sec}$ after finding the peanut. Feeding was interrupted by opening the trap door of the home cage and turning off the lights in the room so that the bird would fly back into its home cage. In phase 2 , the bird was allowed back in to the room where the remainder of the powdered peanut was hidden in the site where it had been found in phase I. A bird with perfect memory should return immediately to the site where it had partially eaten the peanut in phase 1 without looking in any other sites, whereas a bird searching at random without revisiting should find the peanut on average on the fourth visit (Healy and Krebs, 1992). The total number of visits, excluding revisits, (which were very rare) that each bird made to find the peanut was recorded in both phases of each trial. A visit was recorded when the bird perched on the feeder and inspected the contents of the hole by moving the string knot which covered the hole. All individuals were given one trial per day and the order of testing on each day was randomized.

Dissociation trials differed from undissociated trials only in phase II of the experiment. During the retention interval of $20 \mathrm{~min}$, the contents of the baited feeder were removed and the feeder was swapped with one of the other six feeders used in phase I (chosen at random). The feeders were adjusted by covering the holes with string knots, and the bird was allowed back in the room. Thus, in phase II dissociated trials 
differed from undissociated trials in two ways: (1) there was no reward; (2) the spatial location of two of the feeders had been exchanged so that the previously baited feeder had the correct object-specific decoration but incorrect spatial location with respect to phase I of the trial. Therefore, in dissociation trials the bird was forced to chose between the spatial and object-specific cue. The order in which the seven feeders were visited in phase II of each trial was recorded. Birds that relied on spatial information should have returned to the feeder in the correct spatial location first whereas those that relied more heavily on objectspecific cues around the feeder should have looked first in the feeder with the correct object-specific cues. If the birds did not remember which feeder contained peanut in phase $I$ they should search randomly in the seven feeders.

Groups. The experiment was divided into two stages according to age of the birds (Table 1). Two groups of six marsh tits and two groups of six blue tits were tested daily on the one-trial associative memory task in both stages of the experiment. One group of each species was tested on the hidden version ( $\mathrm{MH}$ and $\mathrm{BH}$ ), the other two groups were tested on the visible version of the task (MV and BV). A third group of six birds of each species served as age controls (MC and BC). The two stages were separated by a gap of $56 \mathrm{~d}$, during which the birds were exposed to their appropriate behavioral trials once a week. The gap was included so that the second stage was carried out at the time when wild birds would be at their autumn peak in storing. In order to control for differences in hippocampal volume that might have arisen from the food-storing species storing pieces of nut in their home cages, all six groups $(\mathrm{MH}, \mathrm{BH}, \mathrm{MV}, \mathrm{BV}, \mathrm{MC}$, and $\mathrm{BC}$ ) were maintained on a powdered diet throughout the experiment which consisted of finely ground Orlux, peanut, and sunflower seeds. The ulher two groups of marsh tits, were housed outside in aviaries measuring $1.7 \mathrm{~m}$ wide $\times$ $4.85 \mathrm{~m}$ long $\times 2.55 \mathrm{~m}$ high and were provided with Orlux, pieces of peanut and sunflower seeds which they could store and retrieve during stage I of the experiment. They were then housed indoors in individual cages and in stage II of the experiment they received either the hidden (MSH) or visible (MSV) version of the one-trial associative memory task in stage II. These two groups were given Orlux, pieces of peanut and sunflower seeds rather than finely powdered food in their home cages so that they could continue to store and retrieve caches in their home cages. To control for the effect of eating and handling seeds, all groups of birds were given one piece of peanut and one sunflower seed every day at the end of the daily trials. In addition, all the birds were given five wax moth larvae. Typically, marsh tits begin to store food items once they have eaten two or three seeds and in order to minimize the possibility that the birds might attempt to store, these food items were given individually to each bird at about half-hourly intervals during the day.

In the experienced groups, birds were given a block of twenty acquisition trials followed by a dissociation trial in which the array of feeders was transformed in phase II to test whether the birds preferred to rely on spatial position or object-specific cues to solve the task. Groups $\mathrm{MH}, \mathrm{MV}, \mathrm{BH}$, and $\mathrm{BV}$ received five dissociation trials on day $55,65,75,85,95$ in stage $\mathrm{I}$ and all six experienced groups (MH, MV, $\mathrm{BH}, \mathrm{BV}, \mathrm{MSH}$, and MSV) received three dissociation trials on day 172 , 182,192 in stage II. Between each dissociation trial the birds were given retraining trials to reach criterion and then five undissociated trials . Thus, there were four blocks of undissociated trials in stage I and two blocks in stage II.

Four birds from each group were sacrificed at the end of the experiment (day 192) to test for volumetric differences in the hippocampus. In addition to the 32 brains derived from these treatments, eight marsh tits and three blue tits were sacrificed at day 28 posthatch to provide a baseline measure of relative hippocampal volume before the experiment began. In addition, four wild caught blue tits ( $>1$ year) were housed individually indoors under the same conditions and sacrificed at the end of the experiment. Comparison of these wild-caught birds with controls of the same species allows a test of whether or not there is a deprivation effect resulting from the control treatment.

'lo place the timing of the two experimental stages in the context of the overall life-span of the birds, note that before the first stage (day 35 ) the birds are fully grown but have not reached sexual maturity. By the start of the second stage (day 152), the birds have molted into adult plumage and are fully sexually mature. The two stages cover more than a third of the average expected life-span of an $11 \mathrm{gm}$ bird such as the marsh tit or blue tit.

Neuroanatomical preparations. Brains were treated in an identical way to control for effects of shrinkage. At the end of the experiment, birds were given a lethal intraperitoneal overdose of sodium pentobarbitone and perfused transcardially with heparinized physiological saline followed by Zamboni's solution. The brains were postfixed in Zamboni's solution for seven days. The brains were cut as $15 \mu \mathrm{m}$ coronal sections that had been embedded in polywax. Every tenth section was stained with cresyl violet. The volume of the hippocampal region and the remainder of the telencephalon were traced from the sections using a $10 \times$ photographic enlarger. The traced outlines were digitized using a HIPAD digitizer and the volumes of the hippocampus and telencephalon (minus hippocampus) were computed using the formula for a truncated cone (e.g., Krebs et al., 1989; Clayton and Krebs, 1994c). The ectostriatum was also measured as a control region to test whether or not any volumetric changes occur throughout the telencephalon. All measurements were done blind.

\section{Results}

Performance on the one-trial associative memory task

Number of looks to find the seed in phase 2 in undissociated trials. Performance was measured in terms of the number of sites a bird visited to find the peanut in phase 2 of a trial. For simplicity, Figure 1, $a$ and $b$, show the mean values per group summed across blocks of five trials in the two stages of the experiment for the hidden and visible versions of the task, respectively. Random search is represented by the horizontal line. Performance was compared with random by analyzing the distributions of the number of looks to find the peanut in phase II using the $G$ statistic (see Brodbeck, 1994). The advantage of the $G$ statistic is that the results of numerous $G$ tests can be summed and the degrees of freedom can be partitioned so that the effects of individual birds (heterogeneity $G$ ) can be separated from the overall difference from chance (pooled $G$ ). In both hidden (Fig. $1 a$ ) and visible (Fig. $1 b$ ) treatments birds made significantly fewer visits in phase 2 than expected by chance ( $G$ tests all $p<$ 0.05 for total $G$, heterogeneity $G p>0.05$, and pooled $G p<$ 0.05 ).

Performance in phase 1 should differ between the two treatments because in the visible treatment, birds should fly straight to the feeder to eat peanut whereas in the hidden treatment they should search at random until they find the feeder containing the hidden peanut. In phase 1 of the visible treatment, all three groups differed significantly from random search, most individuals finding the peanut on the first look ( $G$ tests all $p<0.05$ for total $G$, heterogeneity $G p>0.05$, and pooled $G p<0.05$ ). In phase 1 of the hidden treatment, birds did not differ significantly from random expectation ( $G$ tests all $p>0.05$ for total $G$, heterogeneity $G p>0.05$, and pooled $G p>0.05$ ).

The number of looks to find the peanut in phase II decreased over the first twenty acquisition trials for all four experienced groups in stage I [note that some birds did not complete all twenty trials]. Marsh hidden (MH), $r^{2}=0.489, F=11.571, d f$ $=1,16, p<0.01$; blue hidden $(\mathrm{BH}), r^{2}=0.763, F=12.557$, $d f=1,9, p<0.01 ;$ marsh visible (MV), $r^{2}=0.466, F=15.708$, $d f=1,18, p<0.001$; blue visible (BV), $r^{2}=0.731, F=$ $32.607, d f=1,12, p<0.01]$. However, there was no significant increase in performance over the first twenty acquisition trials for the two groups of marsh tits that did not experience the task until stage II (MSH and MSV), and it can be seen from Figure 1 that these two groups made fewer looks to find the peanut in phase II than did the four experienced groups that were tested in stage I, as shown by the first four data points. After the acquisition trials, however, there were no further increases in performance for the subsequent four blocks of control trials in stage I for groups $\mathrm{MH}, \mathrm{BH}, \mathrm{MV}$, and $\mathrm{BV}$ and for the two blocks in stage II for all six experienced groups ( $p>0.5$ in all six cases). 

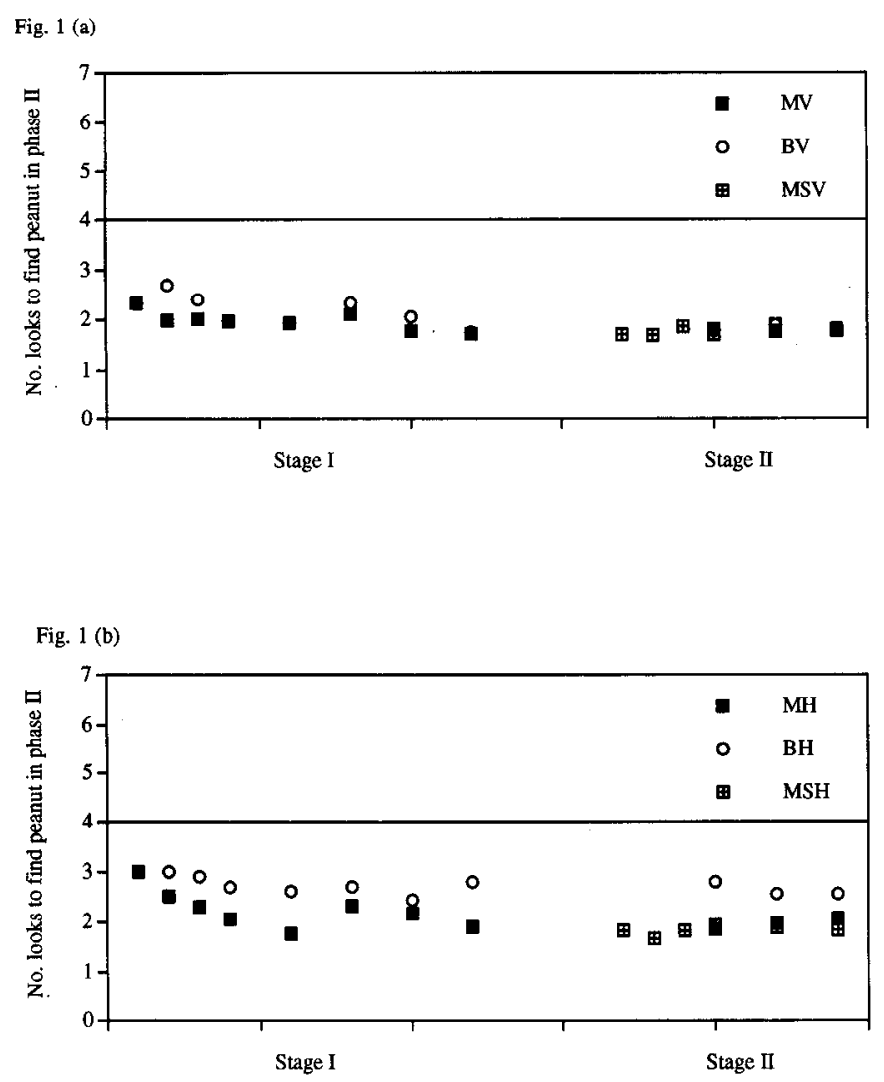

Figure 1. Performance on the one-trial associative memory task for undissociated trials. Mean values summed across blocks of five trials per group of the number of siles visited in phase 2 to find the remaining piece of peanut that had been found in phase 1. $a$, In the "visible" version of the task the peanut was visible in phase 1 and hidden in phase 2. Marsh tits trained and tested on the visible version of the task in both stages of the experiment ( $M V$, solid squares); blue tits trained and tested on the visible version of the task in both stages of the experiment ( $B V$, open circles); marsh tits that had had previous experience of storing and retrieving food and subsequently were trained and tested on the visible version of the task in stage II (MSV, crossed squares). $b$, In the "hidden" version it was hidden in both phases. Marsh tits trained and tested on the hidden version of the task in both stages of the experiment $(M H$, solid squares); blue tits trained and tested on the hidden version of the task in both stages of the experiment ( $\mathrm{BH}$, open circles); marsh tits that had had previous experience of storing and retrieving food and subsequently were trained and tested on the hidden version of the task in stage II (MSH, crossed squares).

In order to examine how the groups differed with respect to overall memory performance, the results were analyzed using an analysis of variance on $\log$ transformations of the data: the mean number of visits to find the seed in phase 2 for each block of undissociated trials in stage I $(n=4)$ and II $(n=2)$ of the experiment formed the dependent variable, and task version (hidden vs visible) split by group (marsh, blue, food-storing marsh) were the independent variables. In both stages of the experiment, there was a significant effect of task version and task $\times$ group (stage I: task version, $F=47.763, d f=1,86 p<$ 0.001 ; task $\times$ group, $F=25.536, d f=1,86, p<0.001$; stage II: task version, $F=13.122, d f=1,59, p<0.001$; task $\times$ group, $F=7.222$, $d f=2,59, p<0.001)$ but no significant effect of trial, group or trial $\times$ group ( $p>0.1$ in each case). This result shows that the task version differed and that the difference between species was more marked in one version than in the other version of the task. Inspection of Figure 1 suggests that these effects arise because marsh tits do better than blue tits (fewer visits to find the seed) in the hidden treatment, irrespective of whether or not they have had experience of storing and retrieving food.

Discrimination between rewarded, unrewarded, and not visited sites in phase 2 in undissociated trials. In order to test the hypothesis that juvenile food-storing species differ from nonstoring species in the way they behave towards "unrewarded" sites, the proportion of the total number of looks each bird made to these three types of site was calculated for each trial in the hidden version of the task. However, since birds may differ in the number of looks they make on different trials, performance based on the total number of looks in phase 2 may bias the results (Shettleworth and Krebs, 1982) because each bird can only visit one "rewarded" site but from zero to several "unrewarded" and "not visited" sites. Thus, for example, a trial in which the seed is found on the first look in phase 2 has a zero probability of visiting unrewarded and not visited sites, while a trial with six looks before finding the seed has greater than zero values for one or both these categories. To take this problem into account, the observed proportions of looks to each type of site were compared with random expectation (see Clayton, 1992b; Clayton and Krebs, 1992).

Figure 2, $a$ and $b$, shows the correctcd proportion of looks to each type of site in phase 2 . For clarity the data presented in the figures are the mean values per group summed across blocks of five trials for the hidden (Fig. $2 a$ ) and visible (Fig. 2b) versions of the task. The proportion of visits to rewarded sites increased gradually over the first 20 acquisition trials for group $\mathrm{MH}$, while the proportion of visits to unrewarded sites gradually decreased. During the first 10 acquisition trials rewarded and unrewarded sites were visited with equal probability. In contrast, the birds in group MSH that had had previous experience of storing and retrieving caches, and were tested on this task 117 d later, made a greater proportion of visits to rewarded sites than to the other two classes of site throughout the acquisition trials.

The data were analyzed with an analysis of variance using arc sin square root transformations of the data: the mean corrected proportion of looks to each type of site for each block of undissociated trials in stage I $(n-4)$ and II $(n=2)$ of the experiment formed the dependent variables in each ANOVA, and task version (hidden vs visible) split by groups (marsh, blue, food-storing marsh) were the independent variables. For rewarded sites, there was a significant effect of task version and task $\times$ groups in (stage I, $F=4.299, d f=1,21, p<0.05$ for task version, $F=9.215, d f=1,21, p<0.01$ for task $\times$ group, and post hoc tests show that group $\mathrm{BH}$ was significantly different from the other five groups, Fisher PLSD, all $p<0.05$. In stage II, $F=3.666, d f=1,30 p<0.01$ for task version, $F=3.527$, $d f=2,30, p<0.05$ for task $\times$ groups) but no significant effect of trial (all $p>0.05$ ). For unrewarded sites, there was a significant difference between the groups on the hidden version of the task (stage I: $F=5.477, d f=1,21, p<0.05$; post hoc tests show that group BH was significantly different from the other two groups, Fisher PLSD, all $p<0.05$; stage II: $F=3.807, d f$ $=2,30, p<0.05)$. For not visited sites, there were no significant effects ( $p>0.05$ for all comparisons).

Of the three groups that were tested on the hidden version of the task (MH, BH, MSH), the following results were obtained. Marsh tits that had experience of food storing and retrieval (MSH) did not differ from marsh tits that had only been tested on the one-trial associative memory task (MH). However, both 

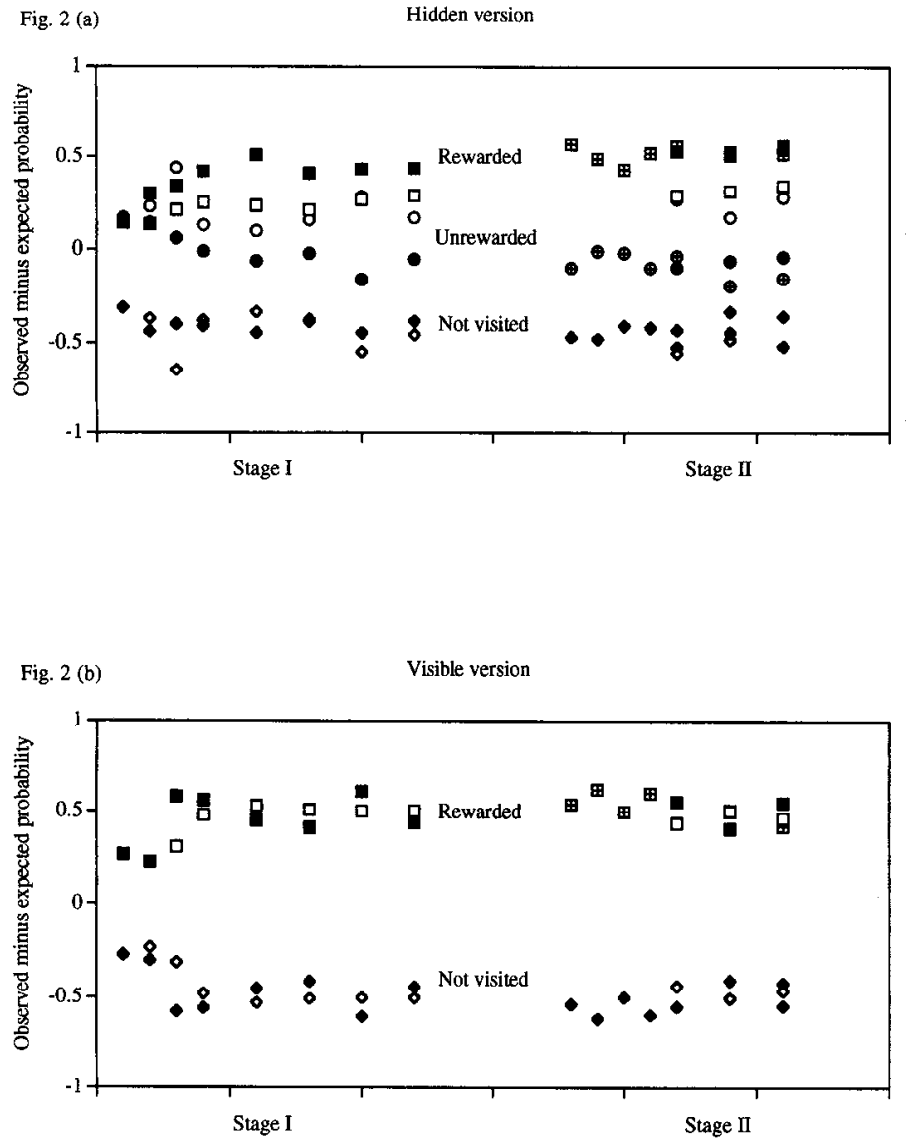

Figure 2. Mean values summed across blocks of five trials per group of the observed minus expected proportion of visits in phase 2 to the threc catcgorics of site, rewarded (squares), unrewarded (circles), and not visited (diamonds), based on behavior in phase 1 of the hidden version of the task (see text for details). $a$, Performance on the hidden version of the task for marsh tits ( $M H$, solid) and blue tits (BH, open) trained and tested on the hidden version of the task in both stages of the experiment and marsh tits that had had previous experience of storing and retrieving food and subsequently were trained and tested on the hidden version of the task in stage II ( $M S H$, crossed $). b$, Performance on the visible version of the task for marsh tits $(M V$, solid) and blue tits ( $B V$, open) trained and tested on the visible version of the task in both stages of the experiment $(B V)$ and marsh tits that had had previous experience of storing and retrieving food and subsequently were trained and tested on the visible version of the task in stage II (MSV, crossed).

groups of marsh tits differed significantly from blue tits $(\mathrm{BH})$ in the way that they discriminated between the classes of sites in stage II: marsh tits make a significantly higher proportion of looks than do blue tits to rewarded sites, a smaller proportion to unrewarded sites, and there is no difference for not-visited sites. Since, the visible version of the task does not test the bird's ability to distinguish between rewarded and unrewarded sites, this species difference in the way they distinguish between categories of site would also explain why marsh tits make fewer looks to find the peanut than do blue tits on the hidden version, but not on the visible version.

Dissociation trials. Memory performance is shown in Figure 3 for each group as the mean percentage of visits to the feeder that was in the correct spatial location and the correct objectspecific cue on the 1st, 2nd, 3rd, 4th, 5th, 6th, and 7th visit (excluding revisits) in phase II of each trial for each of groups MH, MV, BH, and BV (Fig. 3a-d). Figure 4 shows the same analysis for the data in stage II for groups $\mathrm{MH}, \mathrm{MV}, \mathrm{BH}, \mathrm{BV}$, MSH, and MSV (Fig. 4a-f), respectively.

All groups differed significantly from random search, because they tended to visit the feeder in the correct spatial location and the feeder with the correct object-specific cues before visiting the other five feeders ( $G$ tests for both spatial and object-specific cues all $p<0.05$ total; heterogeneity $G$ not significant $p>0.05$; pooled $G$ significant in all cases with $p<0.05$ ). This suggests that all groups remembered both the location and the specific cucs associated with the fecder that had contained peanut in phase I.

Performance in phase II of the dissociation tests was analyzed further by calculating the mean percentage of visits that each bird made to the feeder that was in the correct position on the first visit, correct position on the second visit, correct objectspecific cue on the first visit, correct object-specific cue on the second visit for the two stages of the experiment (five trials in stage I, three trials in stage II). In total there were eight anovas on the $\log$ transformed data, with trial as a repeated measure and species as a between subjects factor. In stage I, there was no significant effect for any of the four anovas (correct position, correct object-specific cues on 1st and 2nd visit, all $p>0.1$ ). This suggests that the birds in all four groups showed an equal preference for the feeder that was in the correct position and the feeder with the correct object-specific cues. The only significant effects were found in stage II for the percentage of visits that each bird made to the feeder that was in the correct position on the first visit (groups, $F=13.2465, d f=2,6, p<0.01$ and post hoc comparisons showed that food-storing marsh tits showed a significantly greater preference to return first to the feeder in the correct position than did blue tits and marsh tits that had not had experience of storing and retrieving food, $p<0.05$, Fisher PLSD).

In order to control for differences in absolute performance between the groups, the data were also analyzed in terms of the number of trials in which each bird preferred to visit the feeder in the correct spatial position before visiting the feeder with the correct object-specific cues. A Kruskal Wallis analysis of variance gave no significant differences between the four groups in stage I $(H=5, d f=3, p>0.1)$ but significant differences between the six groups in stage II $(H=14, d f=5, p<0.05)$. These differences arose because marsh tits that had had no experience of storing and retrieving caches (MH and MV) and blue tits (BH and BV) visited the feeder in the correct spatial position visited before that with the correct object-specific cues on about $50 \%$ of trials; for the other $50 \%$ of trials, the two feeders were visited in the reverse order. In contrast, marsh tits that had had food-storing experience (MSH and MSV) preferred to return to the feeder in the correct spatial location first.

\section{Experience-dependent changes in hippocampal volume}

The results of the volumetric analysis of the brains are illustrated in Figure $5 a-c$ which shows hippocampal volume plotted against telencephalon volume. Following the methods used in previous studies (see Clayton and Krebs, 1994c), volume measurements were logarithmically transformed and analyzed using the residuals of a regression of hippocampal volume on body weight, body weight on telencephalon volume and hippocampal volume on telencephalon volume having removed the effects of body weight (i.e., relative hippocampal volume). A stepwise multiple regression with hippocampal volume as the dependent variable and telencephalon volume and body weight as the independent 


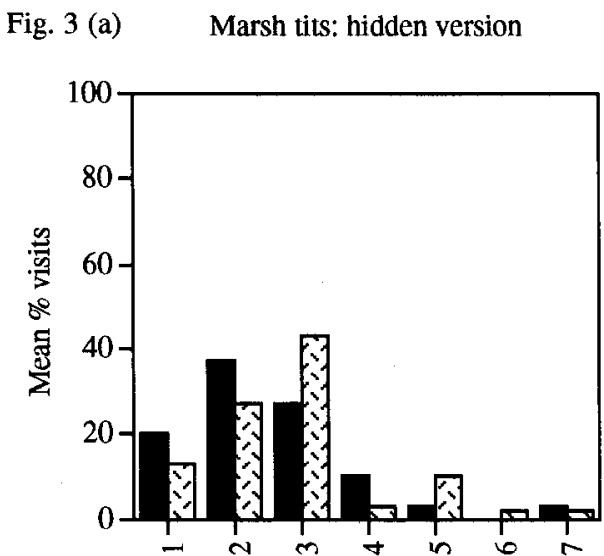

Visit no.

Fig. 3 (b) Blue tits: hidden version

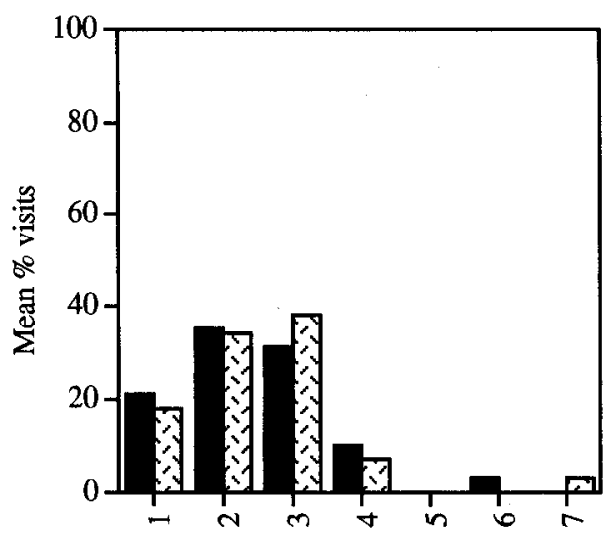

Visit no.
Fig. 3 (c) Marsh tits: visible version

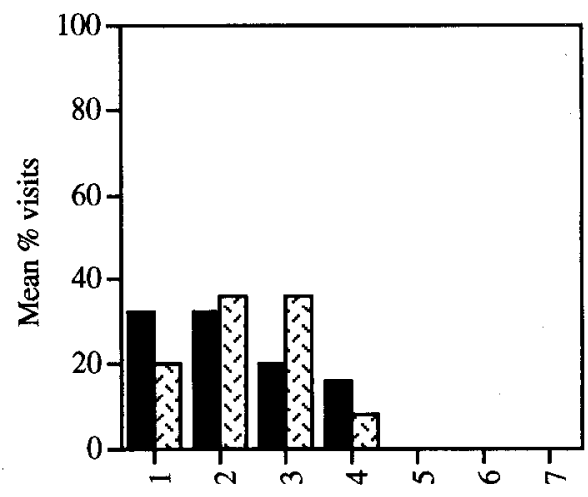

Visit no.

Fig. 3 (d) Blue tits: visible version

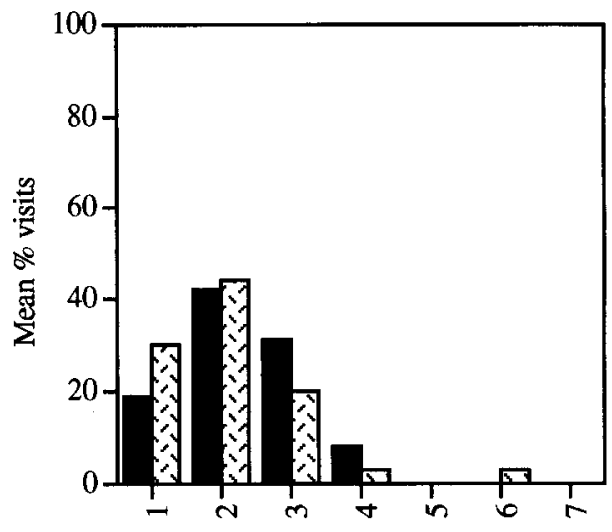

Visit no. variables showed that there was a significant association between telencephalon and hippocampal volume $\left(r^{2}=0.669, F=\right.$ 90.931, $d f=1,45, p<0.001)$ and between body weight and hippocampal volume $\left(r^{2}=0.243, F=14.431, d f=1,45, p<\right.$ $0.01)$.

The residuals of hippocampal volume on telencephalon volume (having removed the effects of body weight) were analyzed by an analysis of variance with relative hippocampal volume as the dependent variable and species as the independent variable. There was a significant difference between the two species at day $192(F=19.624, d f=1,34, p<0.001)$ but not at day 28 $(F=0.371, d f=1,9, p>0.5)$ and it can be seen from Figure $5 a-c$ that this arises because food-storing marsh tits aged $192 \mathrm{~d}$ have relatively larger hippocampal volumes than do blue tits of the same age and day 28 juveniles of either species. In $192 \mathrm{~d}$ old marsh tits, experienced groups show an increase in relative hippocampal volume compared with the control group that had had no experience of either food-storing or the one-trial associative memory task $(F=6.375, d f=4,15, p<0.01)$. Post hoc comparisons using Fisher PLSD show that the difference arises because birds in the control group have significantly smaller relative hippocampal volumes than those of marsh tits in any of the four experienced groups ( $\mathrm{MC}$ vs $\mathrm{MH}, p<0.01$,
MC vs MV, $p<0.01$, MC vs MSH, $p<0.01$, MC vs MSV, $p$ $<0.001)$. There is no difference between marsh tits that had food-storing experience prior to the one-trial associative memory tests and those that did not, nor is there any significant difference between the two versions of the task $p>0.05$ in each case). In $192 \mathrm{~d}$ old blue tits there are no significant differences between the groups $(F=0.845, d f=3,12, p>0.05$ ) which suggests that growth of the hippocampus is not triggered by experience on the one-trial associative memory task in blue tits. Since the wild-caught blue tits did not differ from controls of the same species in relative hippocampal volume, this suggests that deprivation does not affect hippocampal size in blue tits. It also seems unlikely that the smaller size of the hippocampus in control marsh tits can be attributed to an effect of deprivation per se.

The analysis in Figure $5 a-c$ showed that food-storing and onetrial associative memory experience did not induce general growth of the telencephalon, because values were expressed relative to the telencephalon as a whole and because there were no significant differences between the groups in overall telencephalon volume (blue tits, day $192, F=0.358, d f=3,12, p>0.5$; marsh tits, day 192, $F=0.658, d f=4,15, p>0.5$; Juveniles, day $28, F=2.191, d f=1,9, p>0.1)$. The differences arose 
Fig. 4 (a) Marsh tits: hidden version

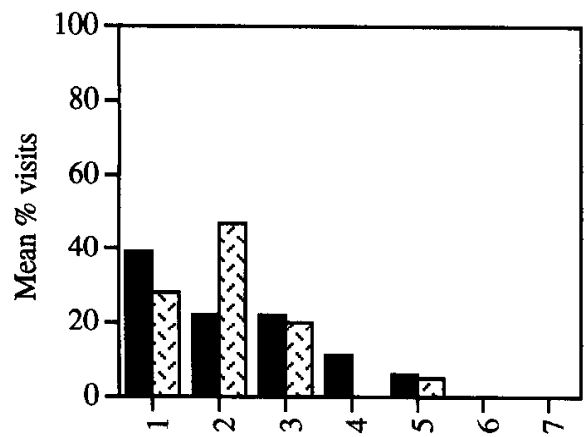

Visit no.

Fig. 4 (b) Blue tits: hidden version

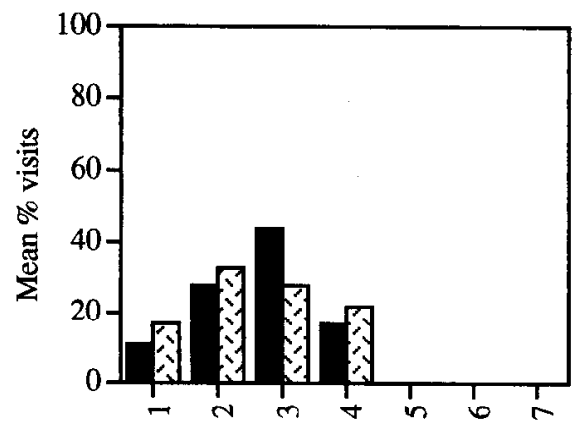

Visit no.

Fig.4 (c) Marsh tit food-storing: hidden version

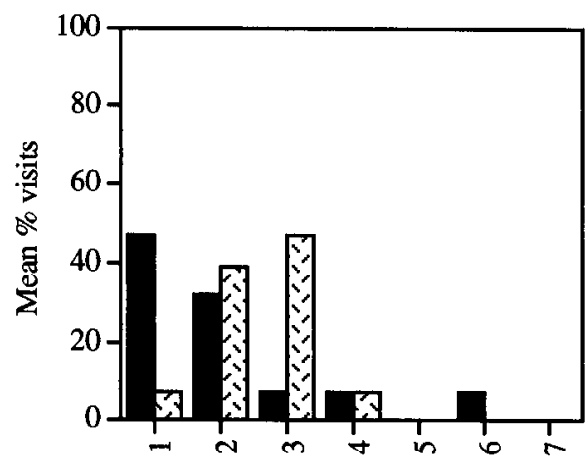

Visit no.
Fig.4 (d) Marsh tits: visible version

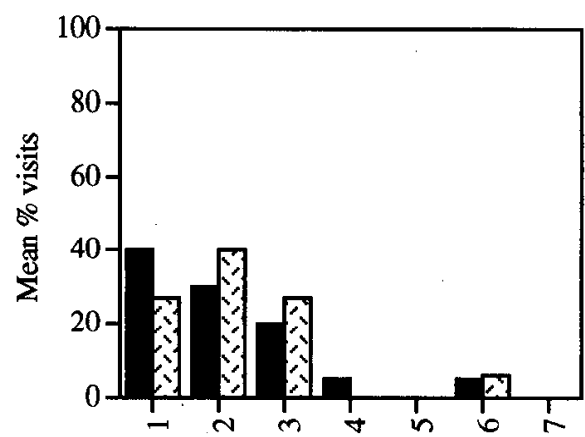

Visit no.

Fig. 4 (e) Blue tits: visible version

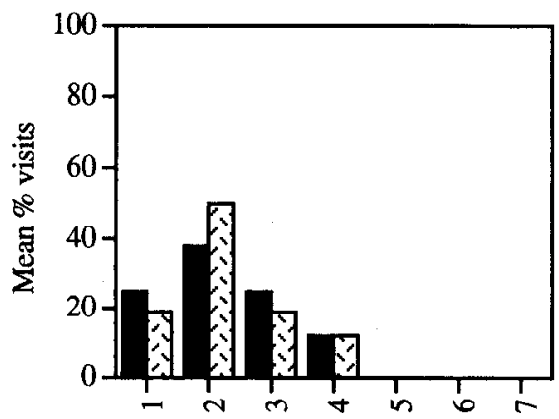

Visit no.

Fig.4 (f) Marsh tit food-storing: visible version

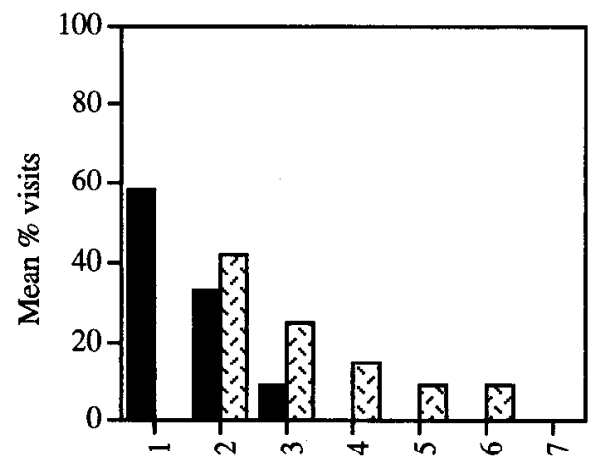

Visit no.
Figure 4. Distribution of visits in the dissociation tests in stage II. Mean percentage of trials per group in which birds visited the feeder that was in the correct spatial location (solid columns) and the correct object-specific cue (hatched columns) on the 1st, 2nd, 3rd, 4th, 5th, 6th, and 7th visit (excluding revisits) in phase II of each trial for stage II of the experiment. Random search is indicated as the horizontal line. $a$, Marsh tits trained and tested on the hidden version of the task $(\mathrm{MH}) ; b$, blue tits trained and tested on the hidden version of the task $(B H) ; c$, marsh tits that had had previous experience of storing and retrieving food and subsequently were trained and tested on the hidden version of the task $(M S H) ; d$, marsh tits trained and tested on the visible version of the task $(M V) ; e$, blue tits trained and tested on the visible version of the task $(B V) ; f$, marsh tits that had had previous experience of storing and retrieving food and subsequently were trained and tested on the visible version of the task $(M S V)$. because experienced marsh tits had larger hippocampal volumes than did control marsh tits, blue tits or day 28 old juveniles of either species (day 28 marsh tits versus blue tits, $F=0.631$, $d f$ $=1,9, p>0.1$; day 192 marsh tits versus blue tits, $F=40.705$, $d f=1,34, p<0.001$; blue tits, day $192, F=0.350, d f=3,12$, $p>0.5$; marsh tits, day $192, F=5.087, d f=4,15, p<0.01$ ).

As a further check of the specificity of the effect on the hippocampal region, an analysis identical to that for the hippocampus was carried out on ectostriatum, a telencephalic nucleus similar in size, and not directly connected, to the hippocampal region (Szekely and Krebs, 1993). Ectostriatum is a visual nu- cleus receiving afferents via the tectofugal pathway, and is considered to be equivalent to the mammalian peristriate cortex (Benowitz, 1982). A stepwise multiple regression with ectostriatum volume as the dependent variable and telencephalon volume and body weight as the independent variables showed no significant association between telencephalon and ectostriatum volume $\left(r^{2}\right.$ $=0.053, F=2.520, d f=1,45, p>0.50$ ) or between body weight and ectostriatum volume $\left(r^{2}=0.063, F=0.181\right.$, df $=$ $1,45, p>0.10)$. The residuals were analyzed by an analysis of variance with relative ectostriatum volume as the dependent variable and species split by age (day 28 vs day 192) as the 
Fig. 5 (a)

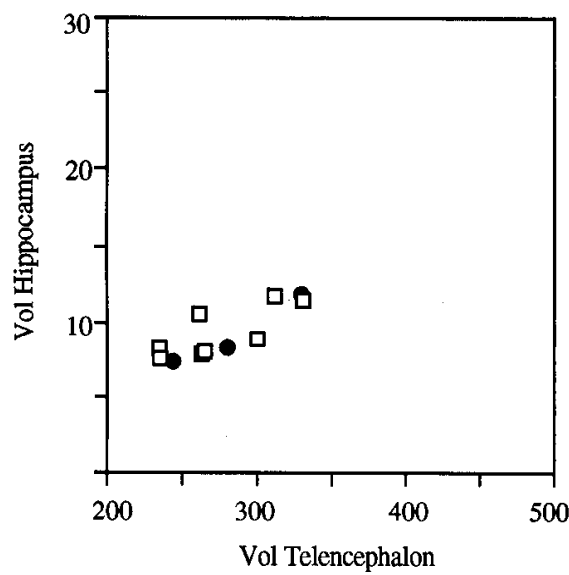

- Juv. Marsh

- Juv. Blue

Fig. 5 (b)

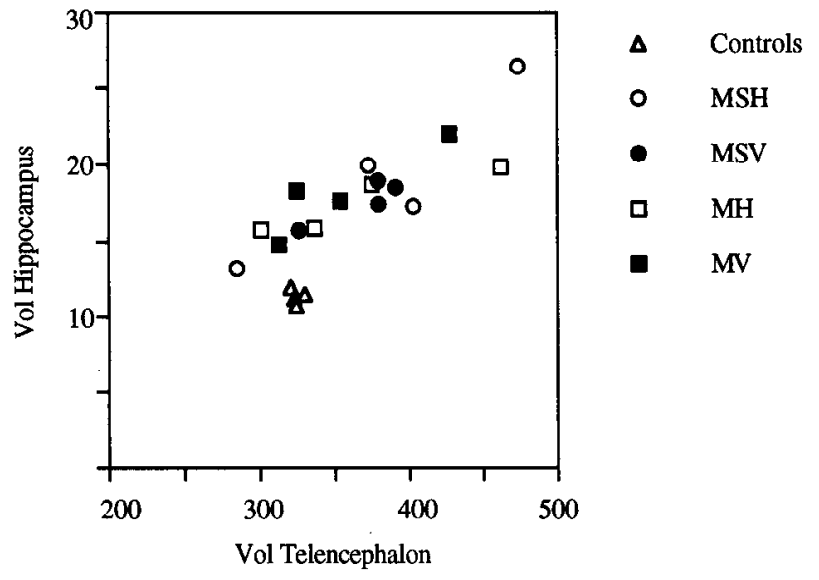

Fig. $5(\mathrm{c})$

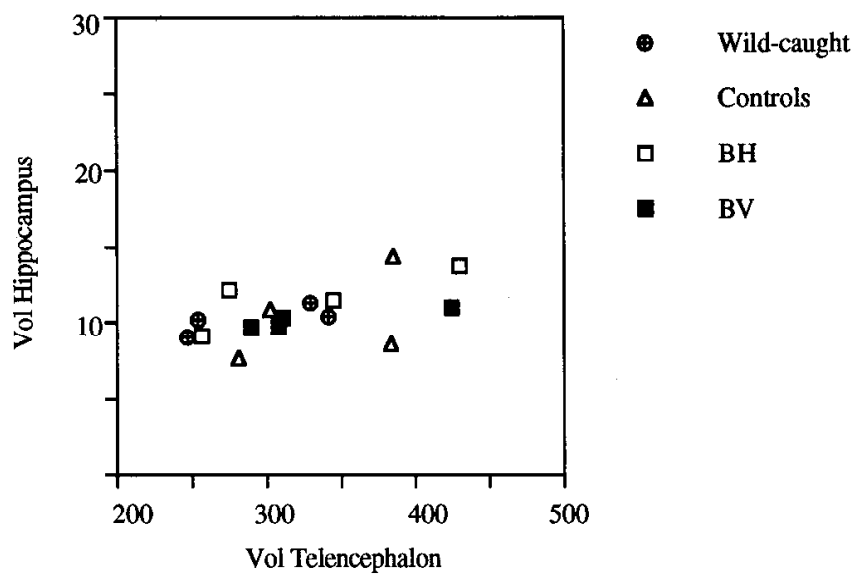

Figure 5. Hippocampal volume plotted against telencephalon volume $\left(\mathrm{mm}^{3}\right)$. a, Juvenile marsh tits (open squares) and blue tits (solid circles). age $28 \mathrm{~d}$ posthatch. $b$, Five groups of marsh tits age $192 \mathrm{~d}$ posthatch: controls (open triangles), marsh tits that were given food-storing ex- independent variable. There was no significant difference in relative ectostriatum volume between the two species at day 28 ( $F$ $=1.124, d f=1,9, p<0.1)$ or at day $192(F=0.303, d f=$ $1,34, p>0.5)$, supporting the conclusion that the effect of experience is specific to the hippocampal region.

\section{Discussion}

Summary of results. The main results for the ontogeny of memory differences between food storers and non-storers were as follows. (1) Performance increased (fewer visits to find the peanut) over the first 20 acquisition trials for all four experienced groups in stage I. However, there was no significant increase in performance for the two groups of marsh tits that did not experience the task until stage II (MSH and MSV), nor any further increase in performance for groups $\mathrm{MH}, \mathrm{BH}, \mathrm{MV}$, and $\mathrm{BV}$ in stage II. The two groups that did not experience the task until stage II and had previously had food-storing experience (MSH and MSV), performed better than groups $\mathrm{MH}, \mathrm{MV}, \mathrm{BH}$, and $\mathrm{BV}$ on the acquisition trials. (2) In undissociated trials, there were no differences between the groups in performance on the visible version of the task. However, marsh tits did better than blue tits in the hidden treatment, irrespective of whether or not they have had experience of storing and retrieving food. This difference arose because marsh tits made a significantly higher proportion of visits than did blue tits to rewarded sites, a smaller proportion to unrewarded sites, and there was no difference for not-visited sites. (3). In dissociation trials, marsh tits with food-storing experience preferentially responded to spatial cues when position and object cues were separated by transforming the array. However, marsh tits that had not had experience of storing and retrieving caches responded equally to both kinds of cue, as did blue tits.

The key results for the ontogeny of volumetric differences between marsh tits and blue tits in brain regions were as follows. (1) Food-storing marsh tits aged $192 \mathrm{~d}$ had relatively larger hippocampal volumes than did blue tits of the same age and day 28 juveniles of either species. (2) In $192 \mathrm{~d}$ old marsh tits, experienced birds had larger relative hippocampal volumes than control birds. There was no difference in relative hippocampal volume of marsh tits that had food-storing experience prior to the one-trial associative memory tests and those that did not, nor was there any significant difference between the two versions of the task on relative hippocampal volume. (3) In $192 \mathrm{~d}$ old blue tits there were no significant differences between the groups. (4) There was no significant difference between species, groups, or task version of the relative volume of the ectostriatum.

Acquisition of memory. The first objective of this study was to test whether the observed differences between adult foodstoring and nonstoring species in performance on the memory tasks are apparent in the juveniles. In stage I, performance increased (fewer visits to find the peanut) over the first 20 acqui-

$\leftarrow$

perience in stage I and trained and tested on either the hidden (MSH, open circles) or visible (MSV, solid circles) versions of the ask in stage II, and marsh tits that were trained and tested on either the hidden ( $M H$ open squares) or visible ( $M V$, solid squares) versions of the one-trial associative memory task both stages of the experiment. $c$, Four groups of blue tits age 192 posthatch: wild-caught adults (crossed circles), controls (open triangles), and blue tits that were trained and tested on either the hidden ( $B H$, open squares) or visible ( $B V$, solid squares) versions of the one-trial associative memory task both stages of the experiment. 
sition trials for all four experienced groups but there was no significant increase in performance for the two groups of marsh tits that did not experience the task until stage II (MSH and MSV). Furthermore, The two groups that did not experience the task until stage II and had previously had food-storing experience (MSH and MSV), performed better than groups $\mathrm{MH}, \mathrm{MV}$, $\mathrm{BH}$, and $\mathrm{BV}$ on the acquisition trials. These results suggests that the ability to remember the location of food encountered previously increases over the first few trials after nutritional independence and that the increase in memory performance follows a similar time course to that of memory for cache sites in that it occurs gradually over a period of about twenty days (Clayton, 1992a, 1994). It has been suggested that food-storing memory increased largely as a result of experience (Clayton, 1994) but it was not possible to distinguish between the effects of maturation (age) and food-storing experience for performance on the one-trial associalive memory lask because groups MSH and MSV differed from groups $\mathrm{MH}$ and MV in two respects: they were both older and had had experience of storing and retrieving their caches.

Species differences in memory. The species differences in performance found in both normal and dissociation trials in this experiment are consistent with those found in previous studies on wild-caught adult marsh tits and blue tits (Clayton and Krebs, 1994a,b). In undissociated trials, there were no differences between the experienced groups in performance on the visible version of the task. However, marsh tits did better than blue tits in the hidden treatment, irrespective of whether or not they have had experience of storing and retrieving food. This difference arose because marsh tits made a significantly higher proportion of looks than did blue tits to rewarded sites, a smaller proportion to unrewarded sites, and there was no difference for not-visited sites. Since, the visible version of the task does not test the bird's ability to distinguish between rewarded and unrewarded sites, this species difference in the way they distinguish hetween categories of site would also explain why marsh tits make fewer looks to find the peanut than do blue tits on the hidden version, but not on the visible version. Thus, the results suggest a difference in memory between the storing and nonstoring species: marsh tits are capable of more subtle discrimination among sites than are blue tits.

The ability to discriminate between rewarded and unrewarded sites does not appear to depend upon food-storing experience since there were no significant differences between the performance of the two marsh tit groups, $\mathrm{MH}$ and $\mathrm{MSH}$, in the blocks of trials in stage II. However, the two groups differed during acquisition trials. It can be seen in Figure 2 that the proportion of visits to rewarded sites increased gradually over the first 20 acquisition trials for group $\mathrm{MH}$, while the proportion of visits to unrewarded sites gradually decreased, and that during the first 10 acquisition trials rewarded and unrewarded sites were visited with equal probability. In contrast, the birds in group MSH that had had previous experience of storing and retrieving caches made a greater proportion of visits to rewarded sites than to other classes of site throughout the acquisition trials. One possible explanation for these results is that the ability to discriminate between rewarded and unrewarded sites increases with age. An alternative explanation is that experience acquired during food-storing may be sufficiently similar to that acquired during training on the one-trial associative memory task so that either type of experience allows development of the ability to preferentially return to the rewarded site. Moreover, it is not known when the volumetric increase in hippocampal volume occurs, but it is possible that the increase in proportion of visits to rewarded sites is associated with an increase in hippocampal volume. Further experiments are required to test when the volumetric increase in hippocampus occurs and whether or not it occurs before or after the onset of food storing.

Food-storing experience and memory. In dissociation trials, marsh tits with food-storing experience preferentially responded to spatial cues when position and object cues were separated by transforming the array, whilst the blue tits responded equally to both kinds of cue, which is consistent with previous experiments comparing adult, wild-caught food-storing and nonstoring species (e.g., Brodbeck, 1994; Clayton and Krebs, 1994a). However, marsh tits that had had no experience of storing and retrieving caches responded equally to both kinds of cue rather than showing the preference for spatial position that is typical of food-storing birds. Given the finding that marsh tits that had had no opportunity to store and retrieve food did not differ from those that had on undissociated trials, it might seem surprising that marsh tits with no food-storing experience behaved more like the nonstorers on the dissociation trials. These results sug gests that the tendency to respond preferentially to spatial cues is acquired as a result of storing and retrieving food. Furthermore, there was no change in preference over the series of dissociation trials in stage I and II which suggests that the preference for spatial cues is experience dependent, rather than maturation or inherent species bias.

One possible adaptive explanation for the emphasis that foodstorers place on spatial position is that object-specific cues are more prone to change (e.g., due to snowfall or dieback of vegetation in winter) between storage and retrieval of food than is spatial location. By relying on spatial position, storers are able to retrieve their hoards even when object-specific cues have changed (Bennett, 1993; Brodbeck, 1994).

It should he noted that the lack of preference for spatial cues does not necessarily mean that the marsh tits that had had no food-storing experience were worse that experienced food storers on spatial tasks. Further experiments are needed to resolve this issue in which birds are tested in a paradigm in which the task can only be solved using spatial cues (Clayton, unpublished observations).

Species differences in relative hippocampal volume. The second objective of this experiment was to test whether or not the volumetric changes in relative hippocampal size could be triggered by training birds on the one-trial associative memory task rather than by experience of food storing and retrieval and, if so, whether or not the changes in hippocampal volume could also be detected in a species that does not store food. The results showed that in the food-storing species, experienced marsh tits aged $192 \mathrm{~d}$ had relatively larger hippocampal volumes than did blue tits of the same age and day 28 juveniles of either species. This is consistent with previous results on the development of the hippocampus in marsh tits and blue tits (Clayton and Krebs, 1994c; Healy et al., 1994).

In the nonstoring blue tits, there were no significant differences between the gruups which suggests that growth of the hippocampus is not experience-dependent in nonstorers. By contrast, in food-storing marsh tits, experienced marsh tits $(\mathrm{MH}$, $\mathrm{MV}, \mathrm{MSH}, \mathrm{MSV}$ ) had larger relative hippocampal volumes than did control birds of the same age that had not been tested on either food storing or the one trial associative memory task. This suggests that hippocampal growth is experience dependent in 
marsh tits but not in blue tits. However, in marsh tits that were trained on the one-trial associative memory test (groups $\mathrm{MH}$, $\mathrm{MV}, \mathrm{MSH}$, and MSV), there was no difference in relative hippocampal volume between birds that had food-storing experience prior to the one-trial associative memory tests and those that did not, nor was there any significant difference between the two versions of the task on relative hippocampal volume. This suggests that the volumetric changes in rclative hippocampal size can be triggered by some aspect of training marsh tits on either version of the one-trial associative memory task. Somewhat surprisingly, birds that were trained on the hidden version of the task did not have relatively larger hippocampal volumes than those trained on the visible version of the task; nor was there any difference in relative hippocampal volume between birds that preferred to return to the correct spatial location in dissociation trials. Further experiments are in progress in order to determine more precisely the nature of the experience required to trigger hippocampal growth.

It has been argued that growth of the hippocampus is associated with some aspect of experience of food-storing (and/or one-trial associative memory) rather than a general effect of enrichment because experience did not induce general growth of the telencephalon and because there was no significant difference in relative volume of the ectostriatum (Clayton and Krebs, 1994c). However, in the study by Clayton and Krebs (1994c) it could be argued that the difference between experienced and control birds was the result of an overall effect of deprivation on controls rather than a lack of food-storing experience. The wild-caught blue tits in this experiment provide an important test of this hypothesis. Since their relative hippocampal volumes did not differ from those of controls of the same species, this suggests that deprivation does not affect hippocampal size in blue tits. Therefore, the smaller size of the hippocampus in control marsh tits is unlikely to be an effect of deprivation per se.

Previous studies have shown that the developing brain of both birds and mammals, including the mammalian hippocampal region, is plastic in response to specific kinds of sensory input or experience and to hormonal influences but the present results are unique in having the following combination of features (Clayton and Krebs, 1994c). First, the effect of experience is independent of age within the range tested, in contrast to, for example, effects of visual experience on the development of the visual cortex in mammals (e.g., see Rauschecker and Marler, 1987). Second, the effect is specific, both in terms of experience (controls and experimentals did not differ in experience other than the specific task of storing and retrieving food) and in terms of the localization of the effect (it is not a general effect as seen in effects of diverse kinds of enrichment on cortical growth in rats; Cramer, 1988). Third, growth of the hippocampus appears to be triggered by some aspect of memory for retrieving previously encountered food in marsh tits but not in blue tits. Although further work is required to test the extent to which this is a general difference between food storers and nonstorers in hippocampal plasticity, the results might be taken to suggest that plasticity in hippocampal volume is an anatomical specialization of the food storer's brain.

\section{References}

Bennett ATD (1993) Spatial memory in a food-storing corvid. I. Near tall landmarks are primarily used. J Comp Physiol, in press.

Benowitz L (1982) Comparative neurology of the telencephalon (Ebbeson SOE, ed). New York: Plenum.

Bingman VP (1990) Spatial navigation in birds. In: Neurobiology of comparative cognition (Olton DS, Kesner RP, eds), pp 423-447, Hillsdale, NJ: Erlbaum.

Bingman VP (1993) In: Vision, brain and behaviour in birds (Zeigler HP, Bischof H-J, eds), pp 132-117. Hillsdale, NJ: Erlbaum.

Brodbeck DR (1994) Memory for spatial and local cues: a comparison of a storing and a non-storing species. Animal Learn Behav 22:119133.

Brodbeck DR, Burack OR, Shettleworth SJ (1992) One-trial associative memory in black-capped chickadees. J Exp Psychol Anim Behav Proc 18:12-21.

Clayton NS (1992a) Ontogenetic development of food-storing and its associated retrieval in marsh tits, Parus palustris. Behaviour 122:1125.

Clayton NS (1992b) Lateralisation and unilateral transfer of spatial memory in marsh tits. J Comp Physiol A 171:807-815.

Clayton NS (1994) The role of age and experience in the behavioural development of food-storing and retrieval in marsh tits, Parus palustris. Anim Behav 47:1435-1444.

Clayton NS (in press) The neuroethological development of memory in food-storing birds: a case of use it, or lose it. Brain Behav Res, in press.

Clayton NS, Krebs JR (1992) Lateralization in Paridae: comparison of a storing and a non-storing species on a one-trial associative memory task. J Comp Physiol A 171:807-815.

Clayton NS, Krebs JR (1994a) One-trial associative memory: comparison of food-storing and non-storing species of birds. Anim I earn Behav, in press.

Clayton NS, Krebs JR (1994b) Memory for spatial and object-specific cues in food-storing and non-storing birds. J Comp Physiol A, in press.

Clayton NS, Krebs JR (1994c) Hippocampal growth and attrition in birds affected by experience. Proc Natl Acad Sci USA, in press.

Cramer CP(1988) Dev Brain Res 42:151-155.

Doupe AJ (1994) Seeds of instruction: hippocampus and memory in food-storing birds. Proc Natl Acad Sci USA 91.

Eichenbaum H, Otto T, Cohen NJ (1992) The hippocampus-what does it do? Behav Neural Biol 57:2-36.

Gaffan D (1974) Recognition impaired and association intact in memory of monkeys after transection of the fornix. J Comp Physiol Psychol 86:1100-1109.

Good M, Macphail EM (1993a) The avian hippocampus and shortterm memory. Q J Exp Psychol, in press.

Good M, Macphail EM (1993b) Hippocampal lesions in pigeons ( $\mathrm{Co}$ lumba livia) disrupt reinforced pre-exposure but not overshadowing or blocking. Q J Exp Psychol, in press.

Erichsen J'I, Bingman VP, Krebs JR (1991) The distribution of neuropeptides in the dorsomedial telencephalon of the pigeon (Columba livia): a basis for regional subdivisions. J Comp Neurol 314:478492.

Hampton RR, Sherry DF (1994) How cache loss affects choice of cache site in black-capped chickadees. Behav Ecol 5:44-50

Healy SD, Krebs JR (1993) Development of hippocampal specialisation in a food-storing bird. Behav Brain Res 53:127-131.

Healy SD, Clayton NS, Krcbs JR (1994) Devclopment of hippocampal specialization in two species of tit (Parus spp.). Behav Brain Res 61: $23-28$.

Krebs JR (1990) Food-storing birds: adaptive specialization in brain and behaviour? Philos Trans R Soc [Biol] 329:55-62.

Krebs JR, Sherry DF, Healy SD, Perry VH, Vaccarino AL (1989) Hippocampal specialization of food-storing birds. Proc Natl Acad Sci USA 86:1388-1392.

Krebs JR, Healy SD, Shettleworth S (1990) Spatial memory of Paridae: comparison of storing and non-storing species $P$. ater and $P$. major. Anim Behav 39:1127-1138.

Krushinskya NL (1966) Some complex forms of feeding behaviour of nutcracker, Nucifraga caryocatactes, after removal of old cortex. $\mathrm{Zh}$ Evol Bioch Fisiol 2:563-568.

Lipp H-P, Scwegeler H, Heinrich B, Cerbone A, Sadile AG (1987) Strain-specific correlations between hippocampal structural traits and habituation in a spatial novelty situation. Behav Brain Res 24:111123.

Jacobs LF, Spencer W (in press) Natural space-use patterns and hippocampal size in kangaroo rats. In press.

Nadel L (1991) The hippocampus and space revisited. Hippocampus $1: 221-229$. 
O'Boyle VJ, Murray EA, Mishkin M (in press) Effects of excitotoxic amygdalo-hippocampal lesions on visual recognition in rhesus monkeys. Soc Neurosci Abstr 19

O'Keefe J, Nadel L (1978) The hippocampus as a cognitive map. Oxford: Clarendon.

Olton D, Kesner R (1990) Neurobiology of comparative cognition. Hillsdale, NJ: Erlbaum.

Raushecker JP, Marler P, eds (1987)Imprinting and cortical plasticity. New York: Wilcy.

Rawlins JNP, Lyford GL, Seferiades A, Deacon RMJ, Cassaday JH (1993) Critical determinants of nonspatial working memory deficits in rats with conventional lesions of the hippocampus or fornix. Behav Neurosei 107:420-433.

Shapiro ML, Olton DS (1994) Hippocampal function and interference. In: Memory systems 1994 (Schacter DL, Tulving E, eds). Cambridge, MA: MIT Press.

Sherry DF, Vaccarino AL (1989) Hippocampus and memory for food caches in black-capped chickadees. Behav Neurosci 103:308-318.
Sherry DF, Vaccarino AL, Buckenham K, Herz RS (1989) The hippocampal complex of food-storing birds. Brain Behav Evol 34:308317.

Sherry DF, Jacobs LF, Gaulin SJC (1992) Spatial memory and adaptive specialization of the hippocampus. Trends Neurosci 17:298-303.

Shettleworth SJ (1990) Spatial memory in food-storing birds. Philos Trans R Soc [Biol] 329:143-151.

Shettleworth SJ, Krebs JR, Healy SD, Thomas CM (1990) Spatial memory of food storing tits: comparisons of memory in storing and non-storing tasks. J Comp Psychol 104:71-81.

Squire LR (1992) Memory and the hippocampus: a synthesis from findings with rats, monkeys, and humans. Psychol Rev 99:195-231.

Squire LR, Zola-Morgan S (1988) Memory, brain systems and behaviour. Trends Neurosci 11:170-175.

Szekely AD, Krebs JR (1993) Target structures of the hippocampal projections in the zebra finch brain. Brain Res Assoc Abstr 10:47.

Vander Wall SB (1990) Food hoarding in animals. Chicago: University of Chicago. 